\title{
Anal Dysplasia Among Patients With Multiple Human Papillomavirus Anal Lesions: Mosaic or Homogeneity?
}

\author{
Emilie Lafferre ${ }^{1}$, Laurent Abramowitz ${ }^{2,3}$, Francine Walker ${ }^{4,5}$, Dalila Benabderrhamanne ${ }^{2}$, Anne Laurain ${ }^{2,3}$, \\ Xavier Duval ${ }^{6,7}$, Florence Tubach ${ }^{8}$ \\ ${ }^{1}$ Polyclinique Reims-Bezannes, Bezannes; ${ }^{2}$ Gastroenterology and Proctology Unit, Bichat University Hospital, Paris; ${ }^{3}$ Ramsay GDS Blomet, \\ Paris; ${ }^{4}$ Université Paris-Diderot, Sorbonne Paris Cité, Laboratoire d'Excellence Inflamex, Faculté de Médecine, Site Bichat, Paris; ${ }^{5}$ Service \\ d'Anatomo-Pathologie, Hôpitaux Universitaires Paris Nord Val de Seine Bichat-Claude-Bernard, Assistance Publique-Hôpitaux de Paris, Paris; \\ ${ }^{6}$ Center of Clinical Investigations, Inserm CIC 1425, Bichat Hospital, Assistance Publique-Hôpitaux de Paris, Paris; ${ }^{7}$ IAME, Inserm U1137, \\ Paris-Diderot University, Paris; ${ }^{8}$ Sorbonne Université, Inserm, Institut Pierre Louis d'Epidémiologie et de Santé Publique, UMR 1136, \\ Assistance Publique-Hôpitaux de Paris, Hôpital Pitié-Salpêtrière, Département Biostatistique Santé Publique et Information Médicale, CIC- \\ 1421, France
}

Purpose: Anal dysplasia is caused by chronic infection with the human papillomavirus and exposes to the risk of anal cancer. The aim of this study was to evaluate the distribution of dysplasia anal grade among patients operated on for multiple anal condylomas with no macroscopic differences.

Methods: This is a cross-sectional study of patients operated on for multiple anal condylomas including a mapping of dysplasia by performing systematically for each patient one biopsy on visible lesion from each of the 4 quadrants on anal margin and in anal canal. All biopsies were read independently by 2 different pathologists.

Results: Among 72 patients, 60 were men and 48 were human immunodeficiency virus (HIV)-infected with a median age of 37.5 years. The proportion of high-grade squamous intraepithelial lesion (HSIL) was higher in the anal canal (41.7\%) compared to the margin $(20.8 \%)(\mathrm{P}=0.004)$. HSIL frequency did not differ according to the quadrant (anterior, posterior, right, and left) of the 2 areas. HSIL on anal canal was not associated with HSIL on anal margin and vice versa $(P=0.390)$. Neither age nor sex was associated to HSIL but HIV positivity increased the risk of HSIL on the anal margin $(\mathrm{P}=0.010)$. Conclusion: Anal dysplasia is heterogeneously distributed in the anal canal as well as between anal canal and anal margin. The diagnostic of the grade of dysplasia for a person should require multiple biopsies on the canal and anal margin.

\section{Keywords: Anus; Neoplasm; Papillomavirus infections}

\section{INTRODUCTION}

Anal cancer is a relatively rare disease accounting for about 30,400 new cases per year worldwide [1]. However, its incidence has dramatically increased in the past decades mainly in human immu-

Received: Apr 1, 2020 • Revised: May 26, 2020 - Accepted: Jun 11, 2020 Correspondence to: Laurent Abramowitz, M.D.

Gastroenterology and Proctology Unit, Bichat University Hospital, 46 rue Henri Huchard, Paris 75877, France

Tel: +33-1-40-25-80-80, Fax: +33-1-40-25-87-83

E-mail: laurent.abramowitz@aphp.fr

ORCID: https://orcid.org/0000-0002-5106-4317

(C) 2021 The Korean Society of Coloproctology

This is an open-access article distributed under the terms of the Creative Commons Attribution NonCommercial License (https://creativecommons.org/licenses/by-nc/4.0) which permits unrestricted noncommercial use, distribution, and reproduction in any medium, provided the original work is properly cited. nodeficiency virus (HIV)-infected patients and particularly among men who have sex with men with an incidence 100-fold higher than in the general population [2].

Human papillomavirus (HPV) anal infection by oncogenic types $(16,18,31,33,35 \ldots)$ is responsible for anal dysplasia. It is not clear whether there is a continuum between low-grade squamous intraepithelial lesion (LSIL), high-grade squamous intraepithelial lesion (HSIL), and cancer, but the strong similarities with cervical HSIL [3] and the high incidence of anal cancer in populations known to have high rates of anal squamous intraepithelial lesions suggest that HSIL is the precursor lesion for anal cancer [4]. For this reason, treatment and screening are more extensive for patients with HSIL. Indeed, if the histological analysis confirmed the presence of HSIL, the lesion is destroyed and very close monitoring is initiated due to the potential risk of canceriza- 
Volume 37, Number 4, 2021

tion.

In clinical practice when a unique macroscopical suspect lesion is identified, targeted biopsy is performed on it. In case of several macroscopically identical lesions, the number of biopsies to perform and their localization are not specified in guidelines. Usually, in the operating room, only 1 or 2 biopsies on randomly chosen visible lesions are performed to classify the grade of dysplasia for the patient. By doing so isn't the grade of dysplasia in some patients under-diagnosed?

In the present study, in performing systematically multiple anal biopsies in patients with macroscopic lesions operated on for multiple anal condylomas, our objective was to assess the dysplasia anal grade repartition.

\section{METHODS}

\section{Patients and study design}

This cross-sectional study was conducted during 6 years (2005 to 2011) in the proctological department of Bichat - Claude-Bernard University Hospital in Paris, France. All consecutive adult patients with multiple and circumferential anal lesion on anal margin and/ or anal canal were proposed to participate to the study. It could be typical condylomas or any anal lesions that may be related to HPV. They were operated on under general anesthesia for electrical coagulation of all HPV-related visible lesion by the same experienced proctologist. For these people with no macroscopic differences among the lesions of the anal area, a biopsy was performed on a macroscopical visible lesion of each of the 4 quadrants (anterior, posterior, right side, and left side) of the anal margin and the anal canal and a histological analysis was performed

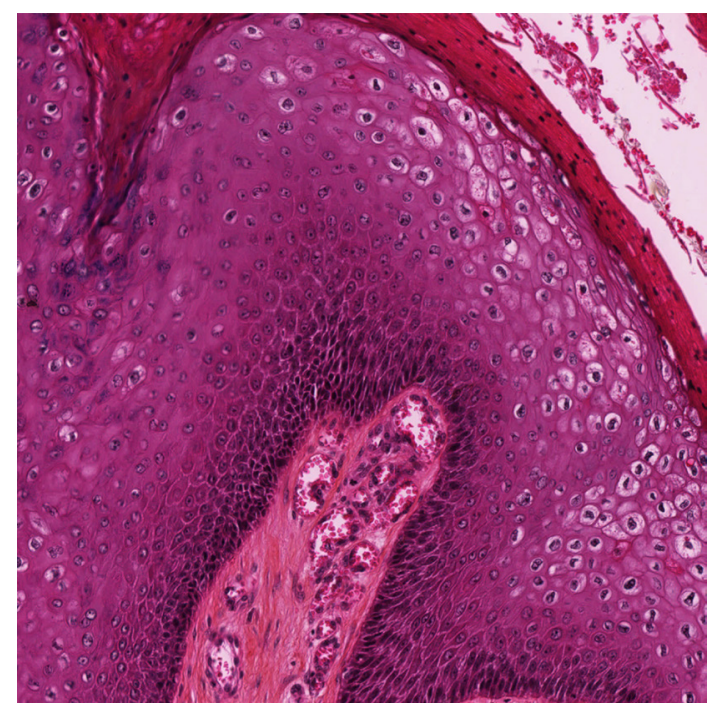

Fig. 1. Representative section of an anal low-grade squamous intraepithelial lesion (anal intraepithelial neoplasia of grade I). H\&E stain, $\times 100$. separately for each biopsy. If no lesion was visible on a quadrant, no biopsy was performed on that quadrant. In case of HPV lesions on external genital organs, they were biopsied and treated at the same time. For each patient, the following data were collected: age, sex, HIV seropositivity, grade of dysplasia on the 4 quadrants of the anal margin and the anal canal and grade of dysplasia on external genital organs.

\section{Histological analysis}

Anal dysplasia levels were diagnosed using 4-mm-thick, paraffinembedded sections of formalin-fixed tissue and stained with hematoxylin and eosin. Diagnosis was based on hyperpapillomatosis associated with hyperacanthosis and the presence of koilocytes. LSIL, also called anal intraepithelial neoplasia of grade I (AIN 1) or low-grade dysplasia, was defined as thickening of the squamous epithelium and slight (lower third) architectural disruption with atypical cells but with no atypical mitosis (Fig. 1). HSIL are also called anal intraepithelial neoplasia, or grade II-III (AIN 2 and 3) or high-grade dysplasia (HSIL) (Fig. 2). AIN 2 was defined as a maturation present in the upper two-thirds of the epithelium and nuclear atypia in both the upper and lower epithelial layers. Mitotic figures are generally confined to the basal two-thirds of the epithelium. AIN 3 (includes intraepithelial carcinoma) corresponds to a maturation absent or confined to the superficial third of the epithelium. Nuclear abnormalities are marked throughout most or all the thickness of the epithelium. Mitotic figures are numerous, often atypical and found at all levels of the epithelium. All biopsies were read independently by a different pathologist, blindly to the result of first analysis.

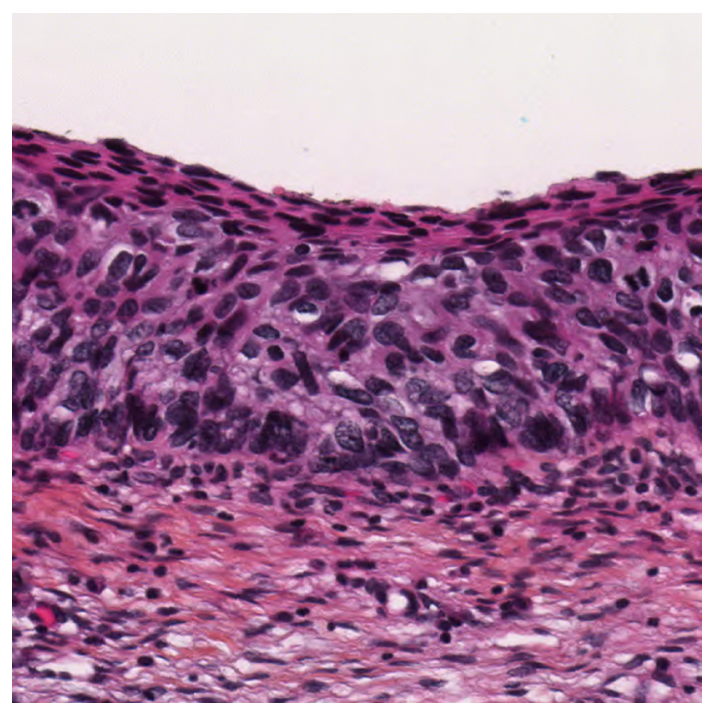

Fig. 2. Representative section of an anal high-grade squamous intraepithelial lesion (anal intraepithelial neoplasia of grade III). H\&E stain, $\times 200$. 


\section{Statistical analysis}

In each quadrant of margin and of anal canal, we classified the quadrant regarding anal dysplasia into 2 categories. The first category was termed "no high-grade dysplasia" included AIN 1, absence of dysplasia and patients who were not biopsied in that quadrant because no visible lesion was observed. The second category termed "high-grade dysplasia" included AIN 2 and AIN 3. The patient was classified as having "high-grade dysplasia" if at least 1 quadrant was classified as high-grade dysplasia. In other cases, the patient was classified as having "no high-grade dysplasia". This classification did not account for lesions on external genital organs.

Categorical variables were described globally or by subgroups (HIV+/HIV-, by quadrant, margin/anal canal and anterior/posterior/left/right) using frequencies and percentages. Their distributions were compared using chi-square tests. Continuous variables were summarized using the median and interquartile range and their distributions were compared between categories using Wilcoxon test. Models of generalized estimating equation taking into account correlated measurement from the different areas for each individual patient were used to test the potential differences in presence of dysplasia between the 4 samples in each area. Univariate analyses (and bivariate analyses adjusted for HIV status) were used to test the potential effect of age on the presence of HSIL. The McNemar test was used to compare rates on paired series. Sensitivity to diagnose a patient having HSIL was assessed for 1,2, or 3 quadrants biopsied, and according to the anal canal or margin location of the biopsies. We have taken as a reference for a sensitivity of $100 \%$ a biopsy of each of the 4 quadrants.

\section{Ethics statement}

All patients received written information before inclusion, signed informed consent, and the local Ethics Committee approved the study (CEERB No IRB0006477).

\section{RESULTS}

\section{Description of the population}

During the study period, 72 patients were prospectively included. Sixty patients (83.3\%) were men, 12 (16.7\%) were women, and 48 (66.7\%, 40 men and 8 women) were HIV-infected. The median

Table 1. Patients' characteristics

\begin{tabular}{lccc}
\hline Characteristic & All & HIV+ & HIV- \\
\hline Patient, No. (\%) & $72(100)$ & $48(66.7)$ & $24(33.3)$ \\
Age (yr) & & & \\
Range & $18-66$ & $24-66$ & $18-58$ \\
$\quad$ Median (IQR) & $37.5(31.0-46.0)$ & $40.5(33.0-47.0)$ & $33.5(27.0-42.5)$ \\
Male sex, No. (\%) & $60(83.3)$ & $40(83.3)$ & $20(83.3)$ \\
\hline
\end{tabular}

HIV, human immunodeficiency virus; IQR, interquartile range. age was 37.5 years (interquartile range, 31.0 to 46.0 years). The proportion of males was identical among HIV-infected or not infected $(83.3 \%)$ but HIV-infected patients were older with a median age at 40.5 (33.0 to 47.0 ) years vs. 33.5 (27.0 to 42.5 ) years $(\mathrm{P}=0.030)$. Patient characteristics are provided in Table 1 .

\section{Anal dysplasia distribution}

On anal margin, HSIL was revealed in 15 of the 72 patients $(20.8 \%)$ and in 30 patients $(41.7 \%)$ in anal canal $(\mathrm{P}=0.004)$. No location (anterior, posterior, right, and left) was more frequently diagnosed with HSIL, neither for anal margin $(\mathrm{P}=0.910)$ nor for anal canal $(\mathrm{P}=0.680)$. Over the entire anus including anal margin and canal, $50.0 \%$ of patients were classified as having HSIL (36 of 72 patients) (Table 2). When considering solely AIN 3 at precancerous lesions, 13 samples were present in 6 male patients; 5 of them with HIV positivity and 1 without.

HSIL on anal canal was not associated with HSIL on anal margin $(\mathrm{P}=0.390)$. Twenty-one out of 57 patients $(36.8 \%)$ had HSIL in the anal canal (at least in one quadrant) in absence of HSIL on the anal margin; 6 out of $42(14.3 \%)$ had HSIL in anal margin in absence of HSIL in anal canal (Table 3).

Eight lesions were detected in 7 patients on external genital organs; 4 on the penis which 2 were HSIL in a man and 4 lesions on the vulva which 3 were HSIL in a woman (Table 2).

With only 1 biopsy on any quadrant of the anal margin, the probability to diagnose a patient having anal HSIL as previously defined was $51.7 \%, 74.5 \%$ with 2 biopsies in 2 different quadrants and $90.0 \%$ with 3 biopsies in 3 different quadrants. In the anal canal, with 1 biopsy probability was $54.2 \%, 75.6 \%$ with 2 biopsies, and $85.0 \%$ with 3 biopsies.

When considering only anal canal biopsy results even in each of the 4 quadrants, $41.7 \%$ of patients (30/72) had HSIL and considering both anal canal and anal margin biopsy results, $50.0 \%$ of patients (36/72) had HSIL. This means that approximately $17 \%$ of patients (6/36) with at least 1 HSIL lesion were missed when con-

Table 2. Anatomical distribution of HSIL according to HIV status

\begin{tabular}{|c|c|c|c|c|c|}
\hline Variable & $\begin{array}{c}\text { All } \\
(n=72)\end{array}$ & P-value & $\begin{array}{c}\text { HIV+ } \\
(n=48)\end{array}$ & $\begin{array}{c}\text { HIV- } \\
(n=24)\end{array}$ & P-value \\
\hline Age, $>50 \mathrm{yr}$ & & & $10(20.8)$ & $2(8.3)$ & 0.150 \\
\hline Male sex & & & 40 (83.3) & 20 (83.3) & $>0.999$ \\
\hline \multicolumn{6}{|l|}{ Patients with HSIL by area } \\
\hline Anal margin & $15(20.8)$ & 0.004 & 14 (29.2) & $1(4.2)$ & 0.010 \\
\hline Anal canal & $30(41.7)$ & & $18(37.5)$ & $12(50.0)$ & 0.200 \\
\hline Anal canal + anal margin & $36(50.0)$ & & $23(47.9)$ & $13(54.2)$ & 0.610 \\
\hline Penis & & & $1(2.1)$ & $0(0)$ & \\
\hline Vulva & & & $1(2.1)$ & $0(0)$ & \\
\hline
\end{tabular}

Values are presented as number (\%).

HSIL, high-grade squamous intraepithelial lesion; HIV, human immunodeficiency virus. 
Table 3. Association between anal canal and anal margin location of HSIL

\begin{tabular}{lccc}
\hline \multirow{2}{*}{ Anal margin } & \multicolumn{2}{c}{ Anal canal } & P-value \\
\cline { 2 - 3 } & No HSIL $(n=42)$ & HSIL $(n=30)$ & \\
\hline No HSIL $(n=57)$ & 36 & 21 & 0.390 \\
HSIL $(n=15)$ & 6 & 9 & \\
\hline
\end{tabular}

Values are presented as numbers of patients.

HSIL, high-grade squamous intraepithelial lesion.

sidering only anal canal biopsies. In HIV-infected patients, biopsies in each of the 4 quadrants of only anal canal allowed diagnosing HSIL in $37.5 \%$ of patients $(18 / 48)$ whereas biopsies performed both on anal margin and in anal canal allowed diagnosing HSIL in $47.9 \%$ of HIV-infected patients (23/48) thereby we might miss $21.7 \%$ of patients $(5 / 23)$ with at least 1 HSIL.

\section{Factor associated with anal HSIL}

Among the 48 HIV-infected patients, 14 (29.2\%) had HSIL on the anal margin while among the 24 non-HIV-infected patients only 1 patient $(4.2 \%)$ had HSIL on this area $(\mathrm{P}=0.010)$. This increasing risk was not observed in the anal canal where HIV infection was not associated to HSIL. Indeed, $18 \mathrm{HIV}$-infected patients had HSIL on anal canal vs. 12 non-HIV-infected patient $(\mathrm{P}=0.200)$. Considering the anal canal and the margin together, the proportion of patients with HSIL did not differ according to HIV status $(\mathrm{P}=0.610)$ nor did the number of HSIL lesion per patient $(\mathrm{P}=0.620)$ (Table 2). Neither age nor sex was associated with HSIL, even when adjusted for HIV status.

\section{DISCUSSION}

This rather large study of patients operated on for multiple condylomas of the anal margin and anal canal in a tertiary proctological Parisian center assessed distribution of HSIL lesions in order to recommend the number and location of biopsies to be sent to the anatomopathologists for evaluation of the risk of HSIL. Every so often considered as simple warts, they were confirmed as dysplasia in $50 \%$ of the cases as we published in other articles [5-8]. The novelty of this study is the heterogeneous location of HSIL in the 2 areas; the anal margin or the anal canal. Indeed, a particular patient may have different grade of dysplasia between the margin and the anal canal. In France many surgeons do not realize several biopsies but often only 1 to evaluate the grade of dysplasia of operated patients. This study shows that, by doing so, only 1 out of 2 HSIL patients can be adequately diagnosed. By repeating biopsies of the margin and the anal canal the probability of positive results dramatically increases and allows the diagnosis of $90 \%$ and $85 \%$ of HSIL patients respectively if 3 samples are taken. The level of monitoring depending on the degree of severity of anal dysplasia, 1 single biopsy may be insufficient to allow the patient to benefit from the appropriate monitoring namely that adapted to his risk of developing anal cancer.

At one time when strategies for early cancer screening are being debated our proposal to simply increase the number of biopsies, when patients are operated for multiple lesions, under general anesthesia seems to be an easy way to obtain a high sensitivity level without morbidity. It may be possible that we did not assess the complete surface of the margin and the anal canal since we did not have a smear on the whole surface of these 2 areas. However, even smears do not have a $100 \%$ sensitivity but rather around $34 \%$ to $69 \%$ according to studies performed in expert sites [9-12]. Moreover, this study evidenced the heterogeneous distribution of HSIL, which would not have been revealed by a smear.

Furthermore with our multiple biopsy-approach during single procedure, we obtained a rate of HSIL of $50 \%$ in our patients, a rate similar to one of the highly-specialized teams in a very highrisk population with anal smear and high-resolution anoscopy (HRA) [13]. Our approach is pragmatic and can be implemented from now, working first on visible lesions. HRA is still far from being of common use due to the time it takes to perform the examination, to get trained on its use (learning curve) and also due to the fact that the exam is not reimbursed (at least in France) while it needs new material with high specific cost. It is thus not possible to recommend its routine use to screen dozen of thousands of patients at risk [14]. Nonetheless, we think that performing this examination for extremely high-risk patients (history of AIN 3 and anal cancer) have to be performed.

Our study shows an increased risk of HSIL on the margin in case of HIV infection. These data are well known [5, 15-19] but what was less expected is the absence of associated risk for anal canal lesions. We can't fully explain this result but it may be that an increased risk of HSIL in anal canal is dominant and overrides the HIV status. One knows how important the level of immunodepression is but that parameter was not collected in this study.

Currently, in France, anal screening by medical physical examination and conventional anoscopy is proposed in the population identified as being at the highest risk [14]; however, recent reviews and international guidelines recommend studies to compare benefits of different strategic approaches to prevent or treat early new anal cancer [20-22].

In our population, we found 6 patients with AIN 3. We would like to think that we helped preventing future cancer in these patients. In reality, the natural history of progression of anal HPV infection to anal cancer is unclear. One study estimated an HSIL regression rate of $23.5 \%$ per year [9] and a recent French study observed a HSIL regression rate of $30 \%$ and healing rate of 33\% after a 3-year follow-up in a cohort of HIV-infected and uninfected patients [23].

In their meta-analysis, Machalek et al. [20] calculated a theorical progression rate from HSIL to anal cancer of 1 in 377 to 1 in 633 patients per year in HIV-positive men and 1 in 4,196 patients per year in HIV-negative men.

We did not demonstrate any relation between sex and HSIL in 
this context of diffuse condyloma. Our study did not take into account the sexual preferences of each patient; however, the aim of the study was not to identify risk factors of anal HSIL that have been already identified in previous studies [5]. Especially since it is now demonstrated that anal HPV infection and AIN may be acquired in absence of anal intercourse in HIV-positive men [18, 19]. Furthermore HSIL risk factors should most probably rather be sought out of virology and molecular biology $[24,25]$ rather than out of standard epidemiological data.

In conclusion, this study demonstrates the heterogeneous anatomical distribution of HSIL lesions. It suggests that blind biopsies can't be the adequate tool to correctly identify patients with at least 1 HSIL lesion, and we consequently recommend performing many biopsies on both anal canal and anal margin at the beginning of surgery for multiple HPV lesions during general anesthesia. Before that recommendation can be formulated, since the natural history of progression of anal HPV infection to anal cancer is unclear, it seems reasonable and pragmatic to consider the destruction of all visible lesions followed by a regular monitoring of patients at risk have a good cost-efficacy ratio given the complexity of the HRA examination and heavy cytological tests.

\section{CONFLICT OF INTEREST}

No potential conflict of interest relevant to this article was reported.

\section{ACKNOWLEDGMENTS}

We thank Dr. Margot Bucau for providing us the histological illustrations.

\section{REFERENCES}

1. Ferlay J, Parkin DM, Curado MP. Cancer incidence in five continents, vol. I to IX. Lyon: IARC Scientific Publications; 2010.

2. Piketty C, Selinger-Leneman H, Bouvier AM, Belot A, MaryKrause M, Duvivier C, et al. Incidence of HIV-related anal cancer remains increased despite long-term combined antiretroviral treatment: results from the french hospital database on HIV. J Clin Oncol 2012;30:4360-6.

3. Melbye M, Sprogel P. Aetiological parallel between anal cancer and cervical cancer. Lancet 1991;338:657-9.

4. Palefsky JM, Holly EA, Ralston ML, Jay N, Berry JM, Darragh TM. High incidence of anal high-grade squamous intra-epithelial lesions among HIV-positive and HIV-negative homosexual and bisexual men. AIDS 1998;12:495-503.

5. Abramowitz L, Benabderrahmane D, Ravaud P, Walker F, Rioux C, Jestin C, et al. Anal squamous intraepithelial lesions and condyloma in HIV-infected heterosexual men, homosexual men and women: prevalence and associated factors. AIDS 2007;21:145765.
6. Abramowitz L, Benabderrahmane D, Walker F, Yazdapanah Y, Yeni P, Rioux C, et al. Determinants of macroscopic anal cancer and precancerous lesions in $1206 \mathrm{HIV}$-infected screened patients. Colorectal Dis 2016;18:997-1004.

7. Schlecht HP, Fugelso DK, Murphy RK, Wagner KT, Doweiko JP, Proper J, et al. Frequency of occult high-grade squamous intraepithelial neoplasia and invasive cancer within anal condylomata in men who have sex with men. Clin Infect Dis 2010;51:107-10.

8. Kreuter A, Siorokos C, Oellig F, Silling S, Pfister H, Wieland U. High-grade dysplasia in anogenital warts of HIV-positive men. JAMA Dermatol 2016;152:1225-30.

9. Tong WW, Jin F, McHugh LC, Maher T, Sinclair B, Grulich AE, et al. Progression to and spontaneous regression of high-grade anal squamous intraepithelial lesions in HIV-infected and uninfected men. AIDS 2013;27:2233-43.

10. Palefsky JM, Holly EA, Hogeboom CJ, Berry JM, Jay N, Darragh TM. Anal cytology as a screening tool for anal squamous intraepithelial lesions. J Acquir Immune Defic Syndr Hum Retrovirol 1997;14:415-22.

11. Nahas CS, da Silva Filho EV, Segurado AA, Genevcius RF, Gerhard R, Gutierrez EB, et al. Screening anal dysplasia in HIV-infected patients: is there an agreement between anal Pap smear and high-resolution anoscopy-guided biopsy? Dis Colon Rectum 2009;52:1854-60.

12. Etienney I, Vuong S, Si-Mohamed A, Flejou JF, Atienza P, Bauer P, et al. Value of cytologic Papanicolaou smears and polymerase chain reaction screening for human papillomavirus DNA in detecting anal intraepithelial neoplasia: comparison with histology of a surgical sample. Cancer 2012;118:6031-8.

13. Wilkin T, Lee JY, Lensing SY, Stier EA, Goldstone SE, Berry MJ, et al. High-grade anal intraepithelial neoplasia among HIV-1-infected men screening for a multicenter clinical trial of a human papillomavirus vaccine. HIV Clin Trials 2013;14:75-9.

14. Liaw YF, Lin DY, Chen TJ, Chu CM. Natural course after the development of cirrhosis in patients with chronic type B hepatitis: a prospective study. Liver 1989;9:235-41.

15. Goldstone SE, Winkler B, Ufford LJ, Alt E, Palefsky JM. High prevalence of anal squamous intraepithelial lesions and squamous-cell carcinoma in men who have sex with men as seen in a surgical practice. Dis Colon Rectum 2001;44:690-8.

16. Palefsky JM, Holly EA, Ralston ML, Jay N. Prevalence and risk factors for human papillomavirus infection of the anal canal in human immunodeficiency virus (HIV)-positive and HIV-negative homosexual men. J Infect Dis 1998;177:361-7.

17. Palefsky JM, Holly EA, Ralston ML, Arthur SP, Jay N, Berry JM, et al. Anal squamous intraepithelial lesions in HIV-positive and HIV-negative homosexual and bisexual men: prevalence and risk factors. J Acquir Immune Defic Syndr Hum Retrovirol 1998;17: 320-6.

18. Piketty C, Darragh TM, Da Costa M, Bruneval P, Heard I, Kazatchkine MD, et al. High prevalence of anal human papillomavirus infection and anal cancer precursors among HIV-infected 
persons in the absence of anal intercourse. Ann Intern Med 2003; 138:453-9.

19. Weis SE, Vecino I, Pogoda JM, Susa JS, Nevoit J, Radaford D, et al. Prevalence of anal intraepithelial neoplasia defined by anal cytology screening and high-resolution anoscopy in a primary care population of HIV-infected men and women. Dis Colon Rectum 2011;54:433-41.

20. Machalek DA, Poynten M, Jin F, Fairley CK, Farnsworth A, Garland SM, et al. Anal human papillomavirus infection and associated neoplastic lesions in men who have sex with men: a systematic review and meta-analysis. Lancet Oncol 2012;13:487-500.

21. Mallari AO, Schwartz TM, Luque AE, Polashenski PS, Rauh SM, Corales RB. Anal cancer screening in HIV-infected patients: is it time to screen them all? Dis Colon Rectum 2012;55:1244-50.

22. Berry JM, Jay N, Cranston RD, Darragh TM, Holly EA, Welton
ML, et al. Progression of anal high-grade squamous intraepithelial lesions to invasive anal cancer among HIV-infected men who have sex with men. Int J Cancer 2014;134:1147-55.

23. Gautier M, Brochard C, Lion A, Henno S, Mallet AL, Bodere A, et al. High-grade anal intraepithelial neoplasia: progression to invasive cancer is not a certainty. Dig Liver Dis 2016;48:806-11.

24. Goldstone SE, Moshier E. Detection of oncogenic human papillomavirus impacts anal screening guidelines in men who have sex with men. Dis Colon Rectum 2010;53:1135-42.

25. Wentzensen N, Follansbee S, Borgonovo S, Tokugawa D, Schwartz L, Lorey TS, et al. Human papillomavirus genotyping, human papillomavirus mRNA expression, and p16/Ki-67 cytology to detect anal cancer precursors in HIV-infected MSM. AIDS 2012;26: 2185-92. 